\title{
Answer to Medical Quiz: Images
}

\section{Answer}

- CT scan of chest shows - A rounded fungal ball in the left lung that is separated from the wall of the cavity by a crescentic halo of air.

- Diagnosis: Aspergilloma

\section{Review}

An aspergilloma is a fungus ball composed of Aspergillus hyphae, fibrin, mucus, and cellular debris found within a pulmonary cavity ${ }^{1}$. Aspergillomas arise in preexisting pulmonary cavities that have become colonized with Aspergillus spp .

Patients are usually asymptomatic but may present with hemoptysis that may be severe.

Patients with classical pulmonary tuberculosis who are left with cavities of $\geq 2 \mathrm{~cm}$ have an approximately 20 percent chance of subsequently developing aspergillomas $^{2}$.
The criteria for the diagnosis of aspergilloma are radiologic evidence of a rounded mass in a pulmonary cavity combined with microbiologic evidence of Aspergillus as the causative agent, usually a positive culture from sputum or detectable Aspergillus $\operatorname{IgG}{ }^{3}$

Asymptomatic cases do not require any treatment. If complicated by hemoptysis surgery, local instillation of amphotericin B or bronchial artery embolisation may be considered.

\section{References}

1. Judson MA, Stevens DA. The treatment of pulmonary aspergilloma. Curr Opin Investig Drugs 2001; 2:1375.

2. Denning DW, Riniotis K, Dobrashian R, Sambatakou H. Chronic cavitary and fibrosing pulmonary and pleural aspergillosis: case series, proposed nomenclature change, and review. Clin Infect Dis 2003; 37 (Suppl 3) :S265.

3. Hope WW, Walsh TJ, Denning DW. The invasive and saprophytic syndromes due to Aspergillus spp. Med Mycol 2005; (43 Suppl 1): S207. 